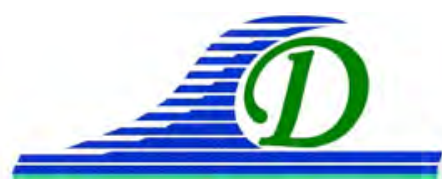

XIII İeses Journées Nationales Génie Côtier - Génie Civil

Dunkerque, 2-4 juillet 2014

DOI:10.5150/jngcgc.2014.005 @ Editions Paralia CFL

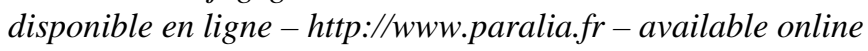

\title{
Formulation analytique du problème de la déformation de la houle en vue d'application numérique
}

\author{
Mohamed CHAGDALI ${ }^{1}$, Soumia MORDANE ${ }^{1}$
}

1. Université Hassan II Mohammedia-Casablanca ; LPPPC FSBM Casablanca Maroc.

m_chagdali@hotmail.com

\section{Communication non présentée}

\section{Résumé :}

Dans le cadre de la théorie potentielle, le problème de la déformation de la houle se ramène à la recherche de potentiel des vitesses et de la forme de la surface libre, qui vérifient l'équation de Laplace dans le domaine fluide et les conditions aux limites sur la surface libre et sur le fond. Les méthodes analytiques de résolution sont basées sur des techniques de perturbation, qui constituent une façon d'obtenir des équations équivalentes au problème de départ. Dans ces méthodes, on utilise un développement en série de perturbation par rapport à un paramètre $\varepsilon$ supposé petit. Pour le problème lié aux non linéarités de la surface libre, le paramètre $\varepsilon$ présente l'ordre de grandeur de l'amplitude sur la longueur d'onde. Dans le cas de l'interaction avec le fond, le paramètre $\varepsilon$ présente l'ordre de grandeur de la pente du fond vis-à-vis des échelles du milieu local et des ondes considérées. En cherchant la solution sous la forme d'une série de perturbation en $(\varepsilon)$, on obtient, à partir du problème de départ, une série de problème aux différents ordres en $(\varepsilon)$ qu'on résout d'une manière séquentielle. La difficulté dans cette démarche est de trouver une solution aux différents ordres supérieurs en $(\varepsilon)$.

Dans ce travail, on montre qu'on peut construire, une formulation récurrente à l'ordre $n$, valable pour $n \geq 2$, sous la forme générale :

$\left.L\left(\varphi_{n}(x, y, z)\right)=S\left(\varphi_{n-1}, \varphi_{n-2}, \ldots \ldots \ldots \ldots . . . . \quad\right)\right)$.

$L_{n}$ est un opérateur linéaire, aux dérivées partielles du second ordre qui correspond à l'ordre $n$ en $(\varepsilon)$. $S$ est un second membre qui ne contient que les informations aux ordres inférieurs à $n$ en $(\varepsilon)$. Cette formulation se prête à la résolution numérique. Une fois les solutions aux ordres $n=0$ et $n=1$ connues, on obtient numériquement les solutions aux différents ordres en inversant une seule fois l'opérateur $L$. Le gain en temps de calcul est important. La résolution numérique se fait par une méthode de résolution classique.

Mots-clés : Houle, Propagation, Méthode semi-numérique. 
Thème 1 - Hydrodynamique côtière 
XIII ${ }^{\text {èmes }}$ Journées Nationales Génie Côtier - Génie Civil Dunkerque, 2-4 juillet 2014 
Thème 1 - Hydrodynamique côtière 
XIII ${ }^{\text {èmes }}$ Journées Nationales Génie Côtier - Génie Civil Dunkerque, 2-4 juillet 2014 
Thème 1 - Hydrodynamique côtière 